\title{
Low prevalence of epilepsy and onchocerciasis after more than 20 years of ivermectin treatment in the Imo River Basin in Nigeria
}

Joseph N. F. Siewe ${ }^{1 *}$ (1), Chinyere N. Ukaga ${ }^{2}$, Ernest O. Nwazor ${ }^{3,4}$, Murphy O. Nwoke², Modebelu C. Nwokeji², Blessing C. Onuoha ${ }^{2}$, Simon O. Nwanjor ${ }^{2}$, Joel Okeke ${ }^{5}$, Kate Osahor ${ }^{2}$, Lilian Chimechefulam², Ann I. Ogomaka², Augustine A. Amaechi', Chika I. Ezenwa², Monika N. Ezike², Chidimma Ikpeama², Ogechi Nwachukwu², Austine I. Eriama-Joseph ${ }^{2}$, Berthram E. B. Nwoke ${ }^{2}$ and Robert Colebunders ${ }^{1}$

\begin{abstract}
Background: High epilepsy prevalence and incidence have been reported in areas with high onchocerciasis transmission. Recent findings suggest that proper community-directed treatment with ivermectin (CDTI) is potentially able to prevent onchocerciasis-associated epilepsy (OAE). We assessed the epilepsy prevalence and onchocerciasis transmission in two Nigerian villages following more than 20 years of CDTI.
\end{abstract}

Methods: A cross-sectional door-to-door survey was performed in two villages in the Imo River Basin reported to be mesoendomic for onchocerciasis (Umuoparaodu and Umuezeala). Individuals were screened for epilepsy using a validated 5-item questionnaire. Persons suspected to have epilepsy were examined by a neurologist or a physician with training in epilepsy for confirmation. Onchocerciasis was investigated via skin snip microscopy and rapid diagnostic tests for Ov16 antibodies. Results were compared with previous findings from the Imo river basin.

Results: A total of 843 individuals from 257 households in the two villages were encountered. We detected four persons with epilepsy (PWE) giving a crude epilepsy prevalence of $0.5 \%$. This finding differs from observations reported 14 years ago which showed an epilepsy prevalence of $2.8 \%$ in the neighbouring village of Umulolo ( $P=0.0001$ ), and $1.2 \%$ from 13 villages in the Imo river basin $(P=0.07)$. The seroprevalence of Ov16 antibodies was found to be $0 \%$. Only $4.6 \%$ of skin snips were positive compared to $26.8 \%$ in previous surveys $(P<0.0001)$. Ivermectin mass distribution coverage in the study sites in 2017 was 79.7\%.

Conclusions: A low epilepsy and onchocerciasis prevalence was observed following more than 20 years of CDTI in the Imo River Basin. Absence of Ov16 antibodies indicates minimal transmission of onchocerciasis. These results contrast with observations from areas of high onchocerciasis transmission, where epilepsy prevalence and incidence remain high. Findings from this study suggest that sustained efforts could eventually achieve elimination of onchocerciasis in these villages.

Keywords: Epilepsy, Onchocerciasis, Nigeria, Ivermectin, Ov16

\footnotetext{
*Correspondence: josephnelson.siewefodjo@uantwerpen.be

${ }^{1}$ Global Health Institute, University of Antwerp, Campus Drie Eiken,

Doornstraat 331, 2610 Wilrijk, Antwerp, Belgium

Full list of author information is available at the end of the article
}

(c) The Author(s). 2019 Open Access This article is distributed under the terms of the Creative Commons Attribution 4.0 International License (http://creativecommons.org/licenses/by/4.0/), which permits unrestricted use, distribution, and reproduction in any medium, provided you give appropriate credit to the original author(s) and the source, provide a link to the Creative Commons license, and indicate if changes were made. The Creative Commons Public Domain Dedication waiver (http://creativecommons.org/publicdomain/zero/1.0/) applies to the data made available in this article, unless otherwise stated. 


\section{Multilingual abstracts}

Please see Additional file 1 for translations of the abstract into five official working languages of the United Nations.

\section{Background}

Epilepsy is a chronic disease estimated to affect 50 million people worldwide according to World Health Organization (WHO) [1]. In general, higher prevalence and incidence of epilepsy are reported from those populations living in low and middle income countries (LMICs) when compared to industrialized countries; the former account for nearly $80 \%$ of the global epilepsy burden [1]. The etiology of epilepsy is very diverse and not yet fully understood. Besides perinatal insults and traumatic injury to the brain, infectious diseases can trigger epilepsy as well. Several parasitic infections have been associated with epilepsy such as cerebral malaria, toxoplasmosis, neurocysticercosis and onchocerciasis [2, 3]. A parasitic etiology of epilepsy implies that many cases could be prevented through timely anti-parasitic treatment.

An epidemiological association between epilepsy and onchocerciasis had already been evoked in Mexico in the 1930s [4] and in Africa (western Uganda) in the early 1990 s $[5,6]$. A case-control study performed in 19911992 in the Mbam Valley in Cameroon prior to community-directed treatment with ivermectin (CDTI) demonstrated a significantly higher microfilarial load in persons with epilepsy than in controls [7]. Study results from other onchocerciasis-endemic African countries underline this association $[2,8]$. To describe this epidemiological phenomenon, the term onchocerciasis associated epilepsy (OAE) was proposed by Kaiser and colleagues [8]. More recently, diagnostic criteria for OAE were proposed [9]. In Tanzania, Uganda and South Sudan, a distinct form of epilepsy has been described as nodding syndrome (NS) $[10,11]$; NS is a debilitating epileptic disorder mainly affecting children between the ages of 3 to 18 years [12]. Case-control studies in northern Uganda and South Sudan focusing on NS patients showed a higher prevalence of onchocerciasis in NS cases compared to non-epileptic controls [13, 14]. It is however unclear how onchocerciasis might cause NS. Johnson et al. suggested that an immunological cross-reaction of onchocerciasis-specific antibodies may provoke a neurotoxic reaction and trigger NS [15]. On the other hand, a cohort study in Cameroon showed a strong dose-response type of association between onchocercal skin microfilariae density as a child and the development of epilepsy later in life [16]. The latter study suggests that the microfilariae themselves are the trigger of the epilepsy. Another phenotypic entity known as the Nakalanga syndrome (epilepsy, stunting, absence or delayed development of external signs of sexual development) also occurs in onchocerciasis-endemic areas [17]. Head nodding seizures and Nakalanga features are increasingly considered as part of the OAE spectrum, alongside other forms of epilepsy [18].

In 2004, following a decade of annual community-directed treatment with ivermectin (CDTI) in the Imo River Basin in Nigeria, a high epilepsy prevalence was observed in villages with high onchocerciasis prevalence [19]. Since 2004, these villages have benefited from another decade of annual CDTI and four years of biannual CDTI. In northern Uganda it was observed that optimal onchocerciasis control was potentially able to stop the incidence of NS and other forms of OAE [20]. Therefore we sought to investigate the effect of more than two decades of CDTI on the prevalence of epilepsy in villages which hitherto were initially meso/hyperendemic for onchocerciasis [21]. We also verified ivermectin coverage and the ongoing transmission risk for onchocerciasis in the area.

\section{Methods \\ Study setting}

The study took place in June 2018 and focused on villages located in the Imo River Basin (Imo State, Nigeria), a known breeding site of the vector Simulium damnosum complex. We selected the Ihite Uboma Local Government Area (LGA), and the Abueke Autonomous Community. From Abueke, we selected the two neighbouring villages closest to the Imo River (Umuezeala and Umuoparaodu), so as to verify possible onchocerciasis transmission risks (Fig. 1). These villages are found within a $40 \mathrm{~km}$ radius of Umulolo village in the Okigwe LGA, which had the highest reported epilepsy prevalence during the previous survey in the Imo river basin [19]. Due to logistical reasons, we were not able to include Umulolo in our survey at this time.

\section{Study population}

The entire population of the selected villages was included in the study. The dominant ethnic group in the area is Ibo. Subsistence farming and petty trading are the main occupations of the people.

\section{Study design}

The study was designed as a community-based cross-sectional survey, following a two-stage approach for epilepsy case identification at village level. We used a door-to-door approach to identify epilepsy cases in the communities. For each village, the local authorities were seen a few weeks before the field survey. The study procedures were explained to them and their collaboration was obtained. Thereafter, sensitization sessions were organized in the villages to prepare the inhabitants for the study. The research team included a 


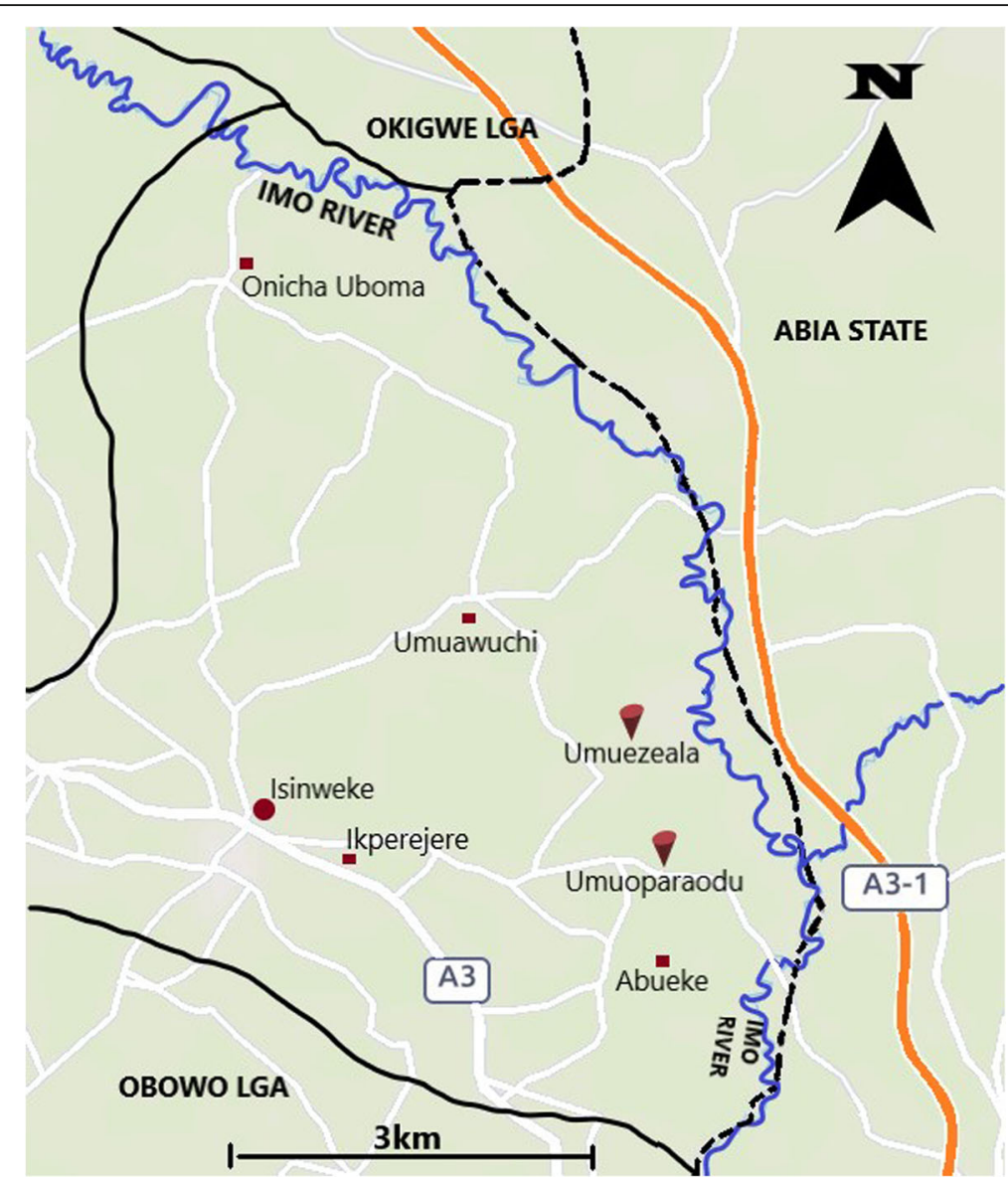

Fig. 1 Map showing the locations of Umuezeala and Umuoparaodu. Distance from the Imo river: Umuezeala $=1.07 \mathrm{~km} ; U$ muoparaodu $=1.17 \mathrm{~km}$

neurologist (EON), ten physicians including one with training in epilepsy (JNFS), parasitologists (CNU), laboratory technicians and public health specialists. We included the local healthcare workers (nurses) and community distributors of ivermectin because they were familiar with the populations and could fluently speak the local Ibo language. All team members were trained on how to do the household survey and ensure confidentiality. The questionnaire was pretested, and was administered primarily in the local language (Ibo) as most individuals did not understand English. In each village, community leaders were interviewed to obtain information pertaining to the villagers' lifestyles, complaints about blackfly bites, their knowledge of onchocerciasis, epilepsy and the emigration/immigration trends over the years.

\section{Household survey}

During the door-to-door survey, we proceeded by first obtaining the signed informed consent of the household head or his representative. The survey was done in two stages. In a first phase, household information was collected which included the ages and sexes of all household occupants, ivermectin treatment status, length of stay of the family in the village, main occupation, notion of previous death of a person with epilepsy and notion of any blind individual in the household. The second part of the family survey consisted of a validated 5-item questionnaire for epilepsy screening [22] which was administered to every member of the household. The five screening questions were: i) history of loss of consciousness with or without loss of bladder control and/or drooling; ii) history of absence or sudden lapse of consciousness of brief duration; iii) history of sudden involuntary jerks of the arms and/or legs (convulsions) lasting for a few minutes; iv) history of sudden and brief bodily sensations, seeing or hearing things that are not there, or smelling strange odours; and v) having been told that he/she had epilepsy or had experienced two epileptic episodes. Family survey data was noted on paper forms.

\section{Epilepsy confirmation}

Anyone with a positive answer to any of the questions during the screening phase was considered as a person suspected to have epilepsy, and was further examined by 
the neurologist/physician with training in epilepsy. The neurological evaluation included both the medical history and a detailed neurological examination to confirm or reject the diagnosis of epilepsy. In case the epilepsy diagnosis was confirmed, the age at the onset of seizures, seizure types and frequencies were noted. Questions were asked concerning potential causes of epilepsy such as birth trauma, head injury, meningo-encephalitis, and cerebral malaria. Data collection by clinicians was done via tablets using the Open Data Kit (https://opendatakit.org). A data manager was responsible for guaranteeing the completeness and good quality of data, and to ensure data transfer from each tablet to the central server for data security. All confirmed cases of epilepsy were referred to the community health centre for proper follow-up.

For quality control, the neurologist (EON) and the physician trained in epilepsy (JNFS) participated in the household visits during the first day of the survey and were thus able to clerk healthy individuals in order to confirm that the latter did not have epilepsy.

\section{Ov16 rapid diagnostic tests}

During the household visits, children aged 6-10 years were identified and referred with their parents/guardians to a workstation to be tested for Ov16 antibodies, using a drop of blood obtained by finger pricking. The children who reported to the workstation came from random areas of the villages and they were all tested. Additionally, persons with confirmed epilepsy and volunteering healthy adults were also tested. The kit used for the tests was the onchocerciasis IgG4 rapid diagnostic test (Ov16 RDT, Standard diagnostics, Inc., Giheung-gu, Yongin-si, Gyeonggi-do, Korea).

\section{Skin snip for onchocerciasis diagnosis}

Participants tested for Ov16 antibodies were also skin snipped for a parasitological diagnosis of onchocerciasis. Two skin snips were obtained from each posterior iliac crest or scapular region using sterilized Holt-type punches. The snips were immediately placed in different wells of a microtitre plate containing three drops of normal saline solution and incubated for $24 \mathrm{~h}$ at room temperature to allow the microfilaria $(\mathrm{mf})$ to emerge into the fluid. After the incubation period, each well was viewed by inverse microscopy using $\times 40$ magnification and microfilariae counted in turns by a trained technician and a parasitologist.

\section{Definitions}

A case of epilepsy was defined as a person who had experienced at least two seizures, unprovoked and without fever, with a minimal time difference of $24 \mathrm{~h}$ between the two events [23].
New cases of epilepsy were defined as cases that appeared within the last 12 months preceding the study period.

Immigrant households were defined as families which were not natives from the study site, but lived in the village.

Onchocerciasis associated epilepsy (OAE) was defined using previously published criteria [9] which included: residence in the village for at least three years, onset of epilepsy between 3 and 18 years, high prevalence of epilepsy in the village, no obvious cause for epilepsy obtained from the medical history and normal psychomotor development prior to the onset of seizures.

Ivermectin coverage referred to the percentage of the entire population that reported taking ivermectin in 2017. The objective was to attain at least $85 \%$ coverage, because it is considered that $15 \%$ of the population are composed of pregnant women, children under five, and the seriously ill who are ineligible for ivermectin treatment [24].

\section{Data management and analysis}

Data was entered into Microsoft Excel 2016 (Microsoft Corporation, Washington, USA) and the Open Data Kit server (ODK Development Team, Washington, USA), and analysed using GraphPad Prism 5 (GraphPad Software, California, USA). For descriptive statistics, continuous data was expressed as means/standard deviations or median/interquartile range (IQR) as appropriate, while categorical data was expressed as percentages. The prevalence of epilepsy was computed by dividing the number of confirmed epilepsy cases by the total number of people registered in the households visited. Past and present prevalences for epilepsy and onchocerciasis were compared using the Chi-squared test at a significance level of $95 \%$.

\section{Ethical considerations}

The study was approved by the ethics committee of the University of Antwerp, Belgium and the ethical committee of the Imo State University, Owerri, Nigeria. All the household heads and individual subjects provided a written informed consent to participate in the study. Parents consented for Ov16 testing in children. In case of illiteracy, an informed verbal consent was given and a thumb print signature was obtained. Information was coded and made anonymous during data entry. The research team was trained to ensure confidential treatment of the data obtained.

\section{Results}

\section{Study population}

A total of 843 individuals were screened for epilepsy in 257 households from the two villages. Table 1 shows the 
Table 1 Characteristics of the study population

\begin{tabular}{|c|c|c|c|}
\hline & Umuezeala & Umuoparaodu & Overall \\
\hline \multicolumn{4}{|l|}{ Households } \\
\hline $\begin{array}{l}\text { Total number of } \\
\text { households }\end{array}$ & 130 & 127 & 257 \\
\hline Native households: $n(\%)$ & $124(95.4 \%)$ & $120(94.5 \%)$ & $243(94.5 \%)$ \\
\hline $\begin{array}{l}\text { Immigrant } \\
\text { households: } n(\%)\end{array}$ & $6(4.6 \%)$ & $7(5.5 \%)$ & $13(5.1 \%)$ \\
\hline $\begin{array}{l}\text { Median household } \\
\text { size: } n \text { (IQR) }\end{array}$ & $3(2-5)$ & $3(2-4)$ & $3(2-4)$ \\
\hline $\begin{array}{l}\text { Family history of death } \\
\text { from epilepsy: } n(\%)\end{array}$ & $6(4.6 \%)$ & $1(0.8 \%)$ & $7(2.7 \%)$ \\
\hline $\begin{array}{l}\text { Median age at death } \\
\text { of PWE in years }\end{array}$ & 19 & 19 & 19 \\
\hline \multicolumn{4}{|l|}{ Participants } \\
\hline Number of individuals & 465 & 378 & 843 \\
\hline Age: median $(\mathrm{IQR})^{\mathrm{a}}$ & $33(19-55)$ & $38(18-55)$ & $36(18-55)$ \\
\hline \multicolumn{4}{|l|}{ Age distribution: $n(\%)^{a}$} \\
\hline $0-9$ years & $52(11.3 \%)$ & 55 (14.6\%) & $107(12.8 \%)$ \\
\hline 10-19years & 70 (15.2\%) & 49 (13.0\%) & $119(14.2 \%)$ \\
\hline $20-29$ years & 79 (17.1\%) & $45(12.0 \%)$ & $124(14.8 \%)$ \\
\hline 30-39 years & $62(13.5 \%)$ & $48(12.8 \%)$ & $110(13.1 \%)$ \\
\hline 40-49 years & $55(11.9 \%)$ & $58(15.4 \%)$ & $113(13.5 \%)$ \\
\hline$\geq 50$ years & $143(31.0 \%)$ & $121(32.2 \%)$ & $264(31.5 \%)$ \\
\hline \multicolumn{4}{|l|}{ Gender: $n(\%)^{\mathrm{b}}$} \\
\hline Female & $233(50.3 \%)$ & $165(44.2 \%)$ & $398(47.6 \%)$ \\
\hline Male & $230(49.7 \%)$ & $208(55.8 \%)$ & $438(52.4 \%)$ \\
\hline
\end{tabular}

IQR Interquartile range, PWE Person(s) with epilepsy; ${ }^{a} 6$ missing data; ${ }^{\text {b } 7}$ missing data

characteristics of the individuals and households surveyed in June 2018. The median household size was 3 (interquartile range, IQR: 2-4). There was a masculine preponderance in the study population, with $52.4 \%$ of male participants versus $47.6 \%$ females and a median age of 36 years (IQR: 18-55). There were 13 immigrant households $(5.1 \%)$ with a mean length of stay in the village of one year. Two blind persons $(0.2 \%)$ were found in the study villages. Seven households $(2.7 \%)$ reported that a family member with epilepsy died in the past at a median age of 19 years; only one person with epilepsy ( $0.1 \%$ of the population) died within the last year. No household reported that a family member developed epilepsy within the 12 months preceding our survey (incidence $=0$ ). Farming and trading were the main activities, being practised by 64.0 and $10.9 \%$ of households respectively.

\section{Prevalence of epilepsy}

A total of 43 individuals answered positively to at least one epilepsy screening question, giving suspected epilepsy prevalence rates of $5.1 \%$. Of these 43 persons suspected to have epilepsy, eight showed up at the consultation desk for confirmation, four were seen in their homes and for the remaining 31 who were unavailable at the time of the survey, the neurologist/physician trained in epilepsy obtained their medical history from family relatives. After thorough history taking for all suspected cases and neurological examination for the available participants, only four out of the 43 persons suspected to have epilepsy met the clinical description of epilepsy, giving a prevalence for confirmed epilepsy of $0.5 \%$. Three $(75 \%)$ of the four persons with confirmed epilepsy met some OAE criteria: their onset of seizures was between 3 and 18 years, there was no obvious cause for the epilepsy and their psychomotor development prior to the onset of seizures had been normal. They were all born and had lived in other onchocerciasis-endemic areas: one in Abuja, one in Katsina and the other in North West Cameroon. They only migrated into the study villages later. No nodding seizures were reported (Table 2) and no person with epilepsy (PWE) was stunted.

The 39 persons suspected to have epilepsy in whom the diagnosis was not confirmed had a median age of 43 years (IQR: 25-60) and had the following differential diagnoses: history of only one seizure, febrile seizures during childhood, non-epileptic tremor, dizziness, dysesthesia and cognitive symptoms such as reduced memory and awareness.

\section{Performance of the epilepsy screening tool}

Of the 43 persons who were suspected to have epilepsy, $32(74.4 \%)$ answered positively to question 1 of the questionnaire, $14(32.6 \%)$ to question $2,19(44.2 \%)$ to question $3,15(34.9 \%)$ to question 4 and $14(32.6 \%)$ to question 5 . When using at least one positive answer to any of the five questions for screening, the positive predictive value for the five questions screening tool was 9.3\% (4/43). When a combination of questions is used for screening, consisting of a positive answer to question 5 (highest specificity during validation [22]) and to any other question, the positive predictive value increased to $28.6 \%(4 / 14)$.

On the first day of the survey, the neurologist/physician trained in epilepsy visited 17 random households in which 51 individuals (6.0\% of the study population) answered negatively to the five questions; all of them were clinically confirmed as not having epilepsy. On this limited sample, the negative predictive value for the 5-question screening tool was $100 \%$. The sensitivity and specificity of the 5 -item questionnaire for epilepsy screening in our study was 100 and $56.7 \%$ respectively (Table 3 ).

\section{Onchocerciasis endemicity and CDTI coverage}

All Ov16 tests from 87 tested participants who reported for testing (52 children aged 6-10 years and 35 
Table 2 Characteristics of confirmed persons with epilepsy

\begin{tabular}{|c|c|c|c|c|}
\hline & & Umuezeala & Umuoparaodu & Overall \\
\hline Number of confirmed PWE & & 3 & 1 & 4 \\
\hline Prevalence of epilepsy & & $0.6 \%$ & $0.3 \%$ & $0.5 \%$ \\
\hline Age of confirmed PWE in 2018: & & $18(16-45)$ & 18 & $18(16.5-38.3)$ \\
\hline Age at onset of epilepsy: Mediar & & $7(6-30)$ & 15 & $11(6.3-26.3)$ \\
\hline Duration of epilepsy in years & & $12(9-15)$ & 3 & $10.5(4.5-14.3)$ \\
\hline Seizure type & Generalized tonic clonic & $3(100 \%)$ & $1(100 \%)$ & $4(100 \%)$ \\
\hline & Absences & $1(33.3 \%)$ & $1(100 \%)$ & $2(50 \%)$ \\
\hline & Focal & 0 & 0 & 0 \\
\hline & Nodding & 0 & 0 & 0 \\
\hline Seizure frequency & Daily: > 30/month & 0 & 0 & 0 \\
\hline & Monthly: > 12/year & $3(100 \%)$ & $1(100 \%)$ & $4(100 \%)$ \\
\hline & Yearly: $<12 /$ year & 0 & 0 & 0 \\
\hline Burns / wounds & & 0 & 0 & 0 \\
\hline Past medical history & Birth dystocia & 0 & 0 & 0 \\
\hline & Cerebral Malaria & 0 & 0 & 0 \\
\hline & Measles & 0 & 0 & 0 \\
\hline & Head trauma & 0 & 0 & 0 \\
\hline & Probable eclampsia & $1(33.3 \%)$ & 0 & $1(25 \%)$ \\
\hline Immigrants & & $2(66.7 \%)$ & $1(100 \%)$ & $3(75 \%)$ \\
\hline Clinical signs of onchocerciasis & Leopard skin & 0 & 0 & 0 \\
\hline & Nodules & 0 & 0 & 0 \\
\hline Seizure treatment & Antiepileptic drugs & $3(100 \%)$ & 0 & $3(75 \%)$ \\
\hline & Traditional medicine & 0 & $1(100 \%)$ & $1(25 \%)$ \\
\hline Ivermectin use prior to seizure o & & $1(33.3 \%)$ & 0 & $1(25 \%)$ \\
\hline Positive Ov16 test & & 0 & 0 & 0 \\
\hline Positive skin snip & & 0 & 0 & 0 \\
\hline
\end{tabular}

IQR: Interquartile Range; PWE: Person(s) with epilepsy

volunteering adolescents/adults) were negative. Four of these 87 participants had positive skin snips (onchocerciasis prevalence: 4.6\%; Median microfilarial load: $2 \mathrm{mf}$ per skin snip [IQR: 1-3]); the age range of skin snip-positive participants was 6-10 years and none of them had ever taken ivermectin. Based on verbal reports of ivermectin use by participants in the households, we calculated the CDTI coverage for the year 2017 to be $79.7 \%$.

Table 3 Sensitivity and specificity of the 5 questions for epilepsy diagnosis

\begin{tabular}{llll}
\hline & & \multicolumn{2}{l}{ Confirmed epilepsy } \\
\cline { 3 - 4 } & & Yes & No \\
\hline Positive screening & Yes & $\mathrm{a}=4$ & $\mathrm{~b}=39$ \\
& No & $\mathrm{c}=0$ & $\mathrm{~d}=51$ \\
\hline
\end{tabular}

Sample population: $N=94$

Sensitivity: $a /(a+c)=100 \%$

Specificity: $d /(b+d)=56.7 \%$

Positive predictive value: $a /(a+b)=9.3 \%$

Negative predictive value: $d /(c+d)=100 \%$

\section{Comparison between previous and current survey}

A survey of 13 villages in the Imo river basin in 2004 revealed an epilepsy prevalence of $1.2 \%$ and average skin snip positivity of $26.8 \%$ [19]. In 2018, the results of our investigation in two villages located in the same area and closest to the Imo river were compared with the 2004 findings. We observed a difference in onchocerciasis prevalence $(26.8 \%$ vs $4.6 \%, P<0.0001)$ and a non-significant difference in epilepsy prevalence $(1.2 \%$ vs $0.5 \%, P=0.07)$ (Table 4$)$. When our findings were compared with the 2004 results from Umulolo Village alone [19] (situated within $40 \mathrm{~km}$ from our study site), differences were observed in the prevalence of both onchocerciasis $(36.0 \%$ vs $4.6 \%, P<0.0001)$ and epilepsy $(2.8 \%$ vs $0.5 \%, P=0.0001)$.

\section{Community leaders' opinions}

Five community leaders were interviewed, two from Umuezeala and three from Umuoparaodu. The highlights of their responses were as follows: (i) Presently, epilepsy is not frequent in the village, and therefore not 
Table 4 Comparison between previous and present surveys

\begin{tabular}{|c|c|c|c|}
\hline & Dozie et al. 2004 [19] & Siewe et al. 2018 & Remarks \\
\hline Study sites & 13 villages in the Imo river basin, Nigeria & $\begin{array}{l}\text { Two villages in the Imo river basin, } \\
\text { Nigeria }\end{array}$ & $\begin{array}{l}\text { The two villages in } 2018 \text { were those } \\
\text { closest to the Imo river }\end{array}$ \\
\hline $\begin{array}{l}\text { Number of } \\
\text { years of CDTI } \\
\text { before the } \\
\text { study }\end{array}$ & 10 years & 24 years & $\begin{array}{l}\text { The } 24 \text { years of CDTI in } 2018 \text { included } \\
\text { four years of biannual distribution }\end{array}$ \\
\hline Methods & $\begin{array}{l}\text { - Door-to-door epilepsy surveys } \\
\text { - Epilepsy screening by asking about previous } \\
\text { epilepsy experience in the households, and } \\
\text { confirmation by a doctor trained in } \\
\text { paediatric neurology } \\
\text { - Skin snip microscopy, no Ov16 testing }\end{array}$ & $\begin{array}{l}\text { - Door-to-door epilepsy surveys } \\
\text { - Epilepsy screening using a 5-item } \\
\text { questionnaire, and confirmation } \\
\text { by a neurologist or a doctor } \\
\text { trained in epilepsy } \\
\text { - Skin snip microscopy and Ov16 } \\
\text { testing }\end{array}$ & $\begin{array}{l}\text { Possible underestimation of epilepsy in } 2004 \\
\text { because only one screening question was } \\
\text { asked, and it is likely that only generalized } \\
\text { convulsive seizures were detected }\end{array}$ \\
\hline $\begin{array}{l}\text { Onchocerciasis } \\
\text { prevalence }\end{array}$ & $26.8 \%$ & $4.6 \%$ & $P<0.0001$ \\
\hline $\begin{array}{l}\text { Microfilaria } \\
\text { density }\end{array}$ & 22.1 per mg of skin snip & 2 per skin snip & Average density from all infected participants \\
\hline $\begin{array}{l}\text { Ov16 RDT } \\
\text { positive results }\end{array}$ & Not done & $0 \%$ & Children and adults were tested in 2018 \\
\hline $\begin{array}{l}\text { Onchocerciasis } \\
\text { endemicity }\end{array}$ & Meso-endemic & Hypo-endemic & Reduced endemicity after 14 years of CDTI \\
\hline $\begin{array}{l}\text { Epilepsy } \\
\text { prevalence }\end{array}$ & $1.2 \%$ & $0.5 \%$ & $P=0.07$ \\
\hline
\end{tabular}

$C D T I$ Community-directed treatment with ivermectin, RDT Rapid diagnostic test

well known by the community. All five interviewees could recall at most one death due to epilepsy in recent years. (ii) Blindness is not common presently as compared to the past. They believed that ivermectin distribution helped to reduce blindness in the community. (iii) There are complaints of insects' bites, mostly near the Imo river and in the farm. The nuisance due to insects has not changed with time. Villagers burned the chaffs from palm nuts so that the smoke should repel insects from their homes. However, they were still frequently bitten by insects while working in the farms. (iv) People do not rear pigs in the villages. (v) There had been more immigration than emigration in the community during the recent years.

\section{Discussion}

For several decades, the Imo River Basin had been a known mesoendemic focus for onchocerciasis, which led to the implementation of annual CDTI in 1994 [19, 21] and biannual CDTI since 2015. Previous reports highlighted a high epilepsy prevalence in this area [19]. Our study in 2018 investigated the onchocerciasis and epilepsy situation after 24 years of CDTI and compared the results with observations made 14 years earlier (2004), when only 10 rounds of annual CDTI had been implemented [19]. The two villages under investigation were close to the Imo river and located within a $40 \mathrm{~km}$ radius of Umulolo, which has a similar ecological environment and had the highest epilepsy prevalence $(2.8 \%)$ in the previous survey [19]. As in the 2004 study by Dozie et al. [19], we used the house-to-house approach which remains the standard for epilepsy surveys [25]. A lower epilepsy prevalence was observed when comparing our findings with those from Umulolo, alongside an almost inexistent transmission of onchocerciasis in 2018.

More than half of the village residents were older than 30 years; this population structure differs from typical African settings which generally have youthful populations [26]. Among other reasons, a possible explanation could be an important immigration rate of adults into the study villages as reported by community leaders. We also noted a low epilepsy-related mortality of $0.1 \%$ suggesting that the PWE are likely to live beyond the age of 30 years.

The fact that most PWE in the 2004 study were older than 20 years could be due to an age shift of PWE from 10 to 19 years to $20-29$ years because of a reduced incidence of OAE after a decade of CDTI, as previously described [27]. In the present survey, three PWE (75\%) were aged $10-19$ years and met some of the OAE criteria. However, they were not born in the study sites and had not received ivermectin prior to seizure onset. They could have been exposed to onchocerciasis in their previous residences and later developed OAE-related seizures either before or after their immigration into the study villages. It is highly probable that effective onchocerciasis control has prevented children from developing epilepsy in the native population. The only PWE in our 
study whose seizures began in adulthood (at the age of 30 years) was a native of Umuezeala who had been receiving ivermectin and had a history of probable eclampsia followed by a non-traumatic coma a few months before the onset of seizures. In addition, the 0\% seroprevalence of Ov16 antibodies coupled with a very low onchocerciasis prevalence reflect minimal onchocerciasis transmission resulting from optimal control measures.

Four (4.6\%) out of 87 participants had positive skin snips but negative Ov16 results. This can be explained by the moderate sensitivity of the rapid test, especially with the very low microfilaria load observed. Indeed, Golden et al. reported up to $11 \%$ (27/248) false negatives when using the Ov16 RDT under laboratory conditions [28].

The sensitivity (100\%) and specificity (56.7\%) of the 5 -question epilepsy screening tool were similar to the values reported during the validation of the questionnaire (95.1 and 65.6\%, respectively) [22]. The positive predictive value was very low because of the low prevalence of epilepsy and possibly the fact that the community had little knowledge about epilepsy. The household participants readily attributed any unusual feeling to epilepsy, and may have answered yes to some of the screening questions even in the absence of seizures. The persons with confirmed epilepsy had a median age of 18 years as compared to 43 years for the persons in whom the diagnosis was suspected but later rejected; the latter presented mostly with non-epilepsy symptoms including signs of psychic senescence and parkinsonism that were most likely related to ageing [29]. We were able to improve the positive predictive value from 9.3 to $28.6 \%$ by using a combination of positive answers for epilepsy screening rather than just one positive answer.

The observed epilepsy prevalence of $0.50 \%$ is similar to the $0.53 \%$ reported in other parts of Nigeria [30] but different from previous findings in the Imo River Basin, and in particular Umulolo Village [19]. Lower epilepsy prevalence and transmission of onchocerciasis in 2018 compared to the 2004 survey, suggests that proper onchocerciasis control may be able to modify the epidemiology of epilepsy. Furthermore, the observed low epilepsy prevalence in villages having low onchocerciasis transmission contrasts with high epilepsy prevalence in meso/hyperendemic areas and further strengthens the hypothesis of an association between the two pathologies.

The responses from the community leaders suggest that blackflies still constitute a nuisance for the population. Despite the presence of the vector, onchocerciasis prevalence and transmission has reduced because proper CDTI has probably rendered their bites non-infective. This scenario differs from what happened in ivermectin-naïve onchocerciasis-endemic villages in the Democratic Republic of Congo, where a decreased transmission of onchocerciasis is thought to result from a progressive reduction of the number of blackflies [31].

Clinically, the main seizure types were generalized seizures (100\% of cases) and absences (50\% of cases). These results differ from those obtained by Osuntokun et al. in Western Nigeria in the 1980s, where a majority of focal seizures were found [30]. In our study, although $75 \%$ of PWE took antiepileptic drugs and 25\% resorted to traditional medicine, all cases still experienced seizures on a monthly basis. Proper care of PWE in these remote villages must be instituted, and this necessitates the training of local non-physician staff in epilepsy care [32].

The strength of this study resides in the community-based methodology which makes the results comparable with previous findings. The use of Ov16 rapid tests in assessing transmission is a novel approach which was shown to be feasible and effective. As major limitation, not all cases were physically present for epilepsy confirmation; but given the advanced age of these suspected cases and with the concise description of their symptoms obtained from family relatives, it is unlikely that they had epilepsy. Finally, we were unable to exclude other causes of epilepsy including neurocysticercosis. But given that pigs were not reared in these communities, it is unlikely that neurocysticercosis is a confounder in the present survey.

\section{Conclusions}

Optimal CDTI in the Imo River Basin has resulted in less onchocerciasis transmission with a concomitant decrease in epilepsy prevalence and incidence. Sustained efforts towards onchocerciasis elimination could potentially affect the epidemiology of epilepsy, as hypothesized by Dozie et al. [19]. Our findings further strengthen the possibility of a causal relationship between onchocerciasis and epilepsy.

\section{Additional file}

Additional file 1: Multilingual abstracts in the five official working languages of the United Nations. (PDF $344 \mathrm{~kb}$ )

\section{Abbreviations}

CDTI: Community-directed treatment with ivermectin; IgG4: Immunoglobulin G4 (antibody); Mf: Microfilariae; OAE: Onchocerciasis associated epilepsy; ODK: Open data kit; PWE: Person(s) with epilepsy; RDT: Rapid diagnostic test

\section{Acknowledgements}

We thank the authorities and the communities of Umuezeala and Umuoparaodu for their collaboration. We are grateful to the Imo State University for the technical support throughout the mission.

\section{Funding}

The study received funding from the European Research Council, Advanced Grant (ERC-2014-ADG), grant No.671055. 


\section{Availability of data and materials}

The datasets used and/or analysed during the current study are available from the corresponding author on reasonable request.

\section{Authors' contributions}

$\mathrm{RC}, \mathrm{CNU}$ and JNFS conceived the study. JNFS, CNU, EON, MON, MCN, BCO, SON, JO, KO, LC, AIO, AAA, CIE, MNE, Cl, and ON collected data in the field. AIEJ managed, secured and transferred all collected data. JNFS analysed the data and wrote the first manuscript. All authors critically read and approved the final manuscript.

\section{Ethics approval and consent to participate}

All participants gave a signed informed consent to participate. For children who were tested for Ov16, parental consent was obtained. This study received the ethical approval of the University of Antwerp, Belgium and the Imo State University, Nigeria.

\section{Consent for publication}

Not Applicable.

\section{Competing interests}

The authors declare no competing interests.

\section{Author details}

'Global Health Institute, University of Antwerp, Campus Drie Eiken, Doornstraat 331, 2610 Wilrijk, Antwerp, Belgium. Imo State University, Owerri, Nigeria. ${ }^{3}$ Federal Medical Centre, Owerri, Nigeria. ${ }^{4}$ Madonna University Teaching Hospital, Elele, Nigeria. ${ }^{5}$ mo State Ministry of Health, Owerri, Nigeria.

Received: 5 September 2018 Accepted: 6 January 2019 Published online: 23 January 2019

\section{References}

1. World Health Organization. Epilepsy Fact sheet. 2018. Available from: http://www.who.int/en/news-room/fact-sheets/detail/epilepsy. Accessed 2018 July 19

2. Kamuyu G, Bottomley C, Mageto J, Lowe B, Wilkins PP, Noh JC, et al. Exposure to multiple parasites is associated with the prevalence of active convulsive epilepsy in sub-Saharan Africa. Garcia HH, editor PLoS Negl Trop Dis 2014; doi: https://doi.org/10.1371/journal.pntd.0002908.

3. Ngugi AK, Bottomley C, Kleinschmidt I, Wagner RG, Kakooza-Mwesige A, AeNgibise $K$, et al. Prevalence of active convulsive epilepsy in sub-Saharan Africa and associated risk factors: cross-sectional and case-control studies. Lancet Neurol. 2013;12:253-63.

4. Jilek-Aall L. Epilepsy and onchocerciasis: pioneering research of Mexican physicians vindicated. Investig En Salud. 2004;6:22-7.

5. Ovuga E, Kipp W, Mungherera M, Kasoro S. Epilepsy and retarded growth in a hyperendemic focus of onchocerciasis in rural western Uganda. East Afr Med J. 1992:69:554-6.

6. Kipp W, Kasoro S, Burnham G. Onchocerciasis and epilepsy in Uganda. Lancet. 1994;343:183-4.

7. Boussinesq M, Pion SD, Ngangue D, Kamgno J. Relationship between onchocerciasis and epilepsy: a matched case-control study in the Mbam Valley, Republic of Cameroon. Trans R Soc Trop Med Hyg. 2002;96:537-41.

8. Kaiser C, Pion SD, Boussinesq M. Case-control studies on the relationship between onchocerciasis and epilepsy: systematic review and meta-analysis. PLoS Negl Trop Dis. 2013. https://doi.org/10.1371/journal.pntd.0002147.

9. Colebunders R, Nelson Siewe FJ, Hotterbeekx A. Onchocerciasis-associated epilepsy, an additional reason for strengthening onchocerciasis elimination programs. Trends Parasitol. 2018;34:208-16.

10. Dowell SF, Sejvar JJ, Riek L, Vandemaele KAH, Lamunu M, Kuesel AC, et al. Nodding Syndrome. Emerg Infect Dis. 2013;19:1374-3.

11. Kipp W, Tukesiga E, Kaiser C, Asaba G, Rubaale T. Nodding syndrome, Western Uganda, 1994. Am J Trop Med Hyg. 2015;93:198-202.

12. World Health Organization. International Scientific meeting on Nodding Syndrome. Kampala, Uganda; 2012. [Accessed 2018 July 25] Available from: www.who.int

13. Tumwine JK, Vandemaele K, Chungong S. Richer M, Anker M, Ayana Y, et al. clinical and epidemiologic characteristics of nodding syndrome in Mundri County, southern Sudan. Afr Health Sci. 2012;12:242-8.
14. Foltz JL, Makumbi I, Sejvar JJ, Malimbo M, Ndyomugyenyi R, Atai-Omoruto $A D$, et al. An epidemiologic investigation of potential risk factors for nodding syndrome in Kitgum District, Uganda. Preux P-M, editor. PLoS One 2013; doi: https://doi.org/10.1371/journal.pone.0066419.

15. Johnson TP, Tyagi R, Lee PR, Lee M-H, Johnson KR, Kowalak J, et al. Nodding syndrome may be an autoimmune reaction to the parasitic worm Onchocerca volvulus. Sci Transl Med. 2017. https://doi.org/10.1126/ scitranslmed.aaf6953.

16. Chesnais CB, Nana-Djeunga HC, Njamnshi AK, Lenou-Nanga CG, Boullé C, ACZ-K B, et al. The temporal relationship between onchocerciasis and epilepsy: a population-based cohort study. Lancet Infect Dis. 2018;18:1278-86.

17. Föger K, Gora-Stahlberg G, Sejvar J, Ovuga E, Jilek-Aall L, Schmutzhard E, et al. Nakalanga syndrome: clinical characteristics, potential causes, and its relationship with recently described nodding syndrome. Ekpo UF, editor PLoS Negl Trop Dis. 2017. https://doi.org/10.1371/journal.pntd.0005201.

18. Colebunders R, Njamnshi AK, Oijen MV, Mukendi D, Kashama JM, Mandro M, et al. Onchocerciasis-associated epilepsy: from recent epidemiological and clinical findings to policy implications. Epilepsia Open. 2017;2:145-52.

19. Dozie INS, Onwuliri COE, Nwoke BEB, Chukwuocha UM, Chikwendu Cl, Okoro I, et al. Onchocerciasis and epilepsy in parts of the Imo river basin, Nigeria: A preliminary report. Public Health. 2006;120:448-50.

20. Colebunders R, Irani J, Post R. Nodding syndrome-we can now prevent it. Int J Infect Dis. 2016;44:61-3.

21. Gemade Ell, Jiya JY, Nwoke BEB, Ogunba EO, Edeghere H, Akoh Jl, et al. Human onchocerciasis: current assessment of the disease burden in Nigeria by rapid epidemiological mapping. Ann Trop Med Parasitol. 1998;92:S79-83.

22. Diagana M, Preux PM, Tuillas M, Ould Hamady A, Druet-Cabanac M. Dépistage de l'épilepsie en zones tropicales: validation d'un questionnaire en Mauritanie. Bull Soc Pathol Exot 1990. 2006;99:103-7.

23. Fisher RS, Acevedo C, Arzimanoglou A, Bogacz A, Cross JH, Elger CE, et al. ILAE official report: A practical clinical definition of epilepsy. Epilepsia. 2014;55:475-82.

24. Burnham G, Mebrahtu T. Review: the delivery of ivermectin (Mectizan ${ }^{\circledR}$ ). Tropical Med Int Health. 2004;9:A26-44.

25. Bharucha N, Odermatt P, Preux P-M. Methodological difficulties in the conduct of Neuroepidemiological studies in low- and middle-income countries. Neuroepidemiology. 2014;42:7-15.

26. United Nations, editor. The demographic profile of African countries. Addis Ababa, Ethiopia: economic commission for Africa; 2016. Available from: https://www.uneca.org/sites/default/files/PublicationFiles/demographic_ profile_rev_april_25.pdf

27. Siewe FJN, Tatah GY, Tabah EN, Ngarka L, Nfor LN, Chokote SE, et al. Epidemiology of onchocerciasis-associated epilepsy in the Mbam and Sanaga river valleys of Cameroon: impact of more than 13 years of ivermectin. Infect Dis Poverty. 2018;7(1):114. https://doi.org/10.1186/s40249018-0497-1.

28. Golden A, Steel C, Yokobe L, Jackson E, Barney R, Kubofcik J, et al. Extended result Reading window in lateral flow tests detecting exposure to Onchocerca volvulus: A new technology to improve epidemiological surveillance tools. Abrams WR, editor PLOS ONE 2013; doi: https://doi.org/10. 1371/journal.pone.0069231.

29. Schott JM. The neurology of ageing: what is normal? Pract Neurol. 2017;17: 172-82.

30. Osuntokun BO, Adeuja AO, Nottidge VA, Bademosi O, Olumide A, lge O, et al. Prevalence of the epilepsies in Nigerian Africans: a community-based study. Epilepsia. 1987;28:272-9.

31. Lenaerts $E$, Mandro M, Mukendi D, Suykerbuyk P, Dolo $H$, Wonya'Rossi $D$, et al. High prevalence of epilepsy in onchocerciasis endemic health areas in Democratic Republic of the Congo. Infect Dis Poverty. 2018. https://doi.org/ 10.1186/s40249-018-0452-1.

32. Njamnshi AK. Nonphysician management of epilepsy in resource-limited contexts: roles and responsibilities. Epilepsia. 2009;50:2167-8. 